\title{
Reduction in molecular diagnostics of myeloproliferative neoplasms during the COVID-19 pandemic
}

\author{
Stephen E. Langabeer ${ }^{1}$ (D
}

Received: 26 May 2020 / Accepted: 2 July 2020 / Published online: 8 July 2020

(C) Royal Academy of Medicine in Ireland 2020

Dear Sir,

During the current COVID-19 pandemic (caused by severe acute respiratory syndrome coronavirus 2 (SARS-CoV-2)), the focus of many nations' healthcare services has been on the treatment of infected patients and implementation of measures to prevent infection spreading through the community at large. Due to the gravity of the pandemic, other areas of healthcare such as cancer diagnosis and treatment may be negatively impacted. The classical Philadelphia chromosome-negative myeloproliferative neoplasms (MPN) of polycythaemia vera (PV), essential thrombocythaemia (ET), and primary myelofibrosis (PMF) are pathologically related malignancies characterised by an expansion of mature haematopoietic cells, a propensity for thrombosis and haemorrhage, and the ability to transform into acute myeloid leukaemia. The most commonly acquired somatic mutation in these MPN is the JAK2 V617F, present in $95 \%$ of PV patients and in $50-60 \%$ of patients with ET and PMF [1]. An initial study in China found that patients with haematological malignancies had more severe COVID-19 and a higher fatality rate than a control group [2]. In order to assess any impact of the COVID-19 pandemic on the frequency of testing and JAK2 V617F positivity rate, an audit was performed by a referral centre for the molecular diagnostics of haematological malignancies.

The number of requests for the JAK2 V617F was recorded at twenty-five weekly intervals from the beginning of the year 2020 (week 1 commencing on the 6th of January to week 25 commencing on the 22nd of June). JAK2 V617F mutations were detected by an allele-specific PCR approach unchanged throughout the audit period with the laboratory continuing a "business as usual" approach. A reduction in both the number of requests (Fig. 1) and newly

Stephen E. Langabeer

slangabeer@stjames.ie

1 Cancer Molecular Diagnostics, St. James's Hospital, Dublin, Ireland detected patients is apparent after lockdown restrictions for COVID-19 were introduced at the end of week 10. For the first 10 weeks of the year, 403 requests were received (40.3 per week) of which 53 were positive resulting in a mean of 5.3 new cases of JAK2 V617F-positive MPN per week. This level is in keeping with data from the same period in 2019. In the following 15 weeks, corresponding to the period of ongoing COVID-19 restrictions, 289 requests were received of which 46 were positive resulting in a reduced mean of 3.1 new cases of JAK2 V617Fpositive MPN per week. In week 25, a return to normal requesting patterns was observed with six new patients identified from 36 JAK2 V617F requests.

This brief but informative audit describes the impact of the COVID-19 pandemic on the molecular diagnosis of MPN patients with a projected thirty patients not molecularly identified in the initial 15-week period of national COVID-19 restrictions. The reasons for this reduction in test requests are not immediately apparent though it cannot be attributed to a lack of an available service. Delays in the diagnosis of an MPN have previously been shown to result in an increase in potentially preventable thrombotic complications [3]. Followup of MPN patients with a potentially delayed diagnosis is planned. Data is beginning to emerge on the similar impact of the COVID-19 pandemic upon the molecular diagnosis of other cancers [4]. Hopefully, patient attendance and JAK2 $\mathrm{V} 617 \mathrm{~F}$ testing referral from regional haematology services is likely to improve in the forthcoming months with potential for the (albeit delayed) diagnosis of MPN enabling appropriate therapeutic intervention.

\section{Compliance with ethical standards}

This non-interventional, observational study was performed under routine standard of care procedures in accordance with the 1964 Helsinki declaration and its later amendments.

Conflict of interest The author declares that he has no conflict of interest. 


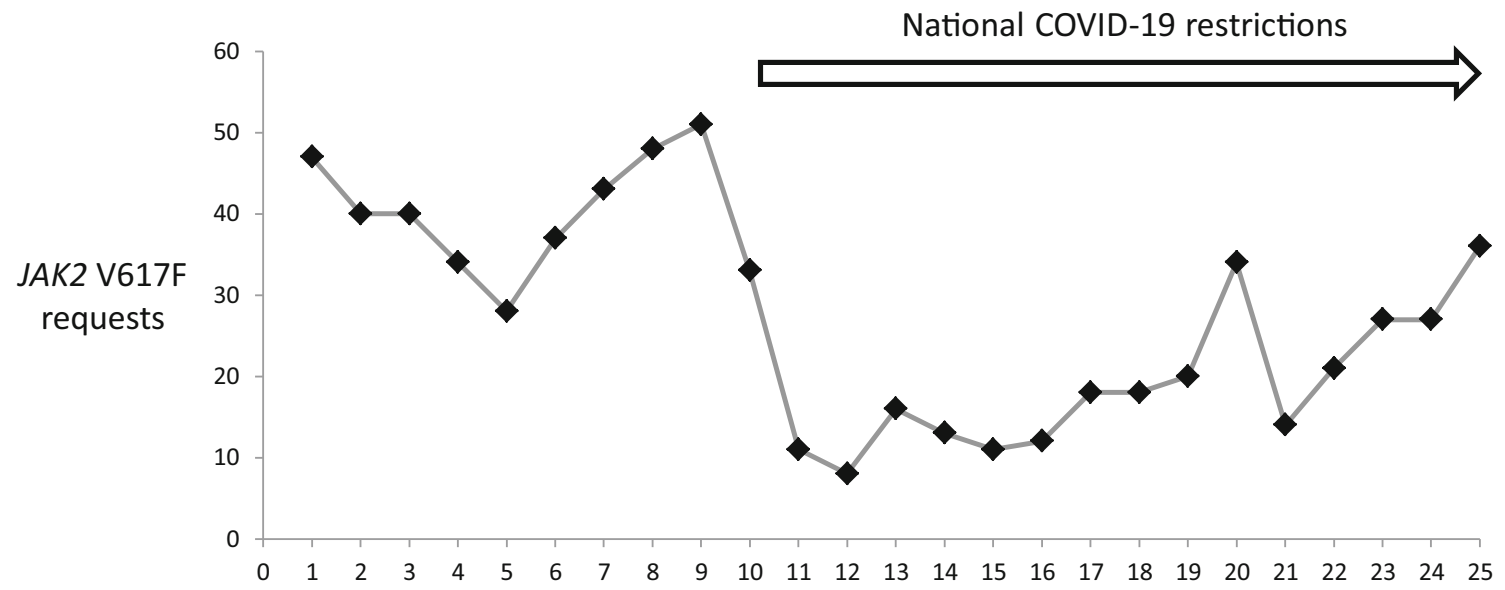

Weeks in 2020

Fig. 1 Weekly incidence of diagnostic JAK2 V617F requests

Informed consent Informed consent was obtained from participants at the referring centres.

\section{References}

1. Langabeer SE, Andrikovics H, Asp J, Bellosillo B, Carillo S, Haslam K, Kjaer L, Lippert E, Mansier O, Oppliger Leibundgut E, Percy MJ, Porret N, Palmqvist L, Schwarz J, McMullin MF, Schnittger S, Pallisgaard N, Hermouet S, the MPN\&MPNr-EuroNet (2015) Molecular diagnostics of myeloproliferative neoplasms. Eur J Haematol 95:270-279

2. He W, Chen L, Chen L, Yuan G, Fang Y, Chen W, Wu D, Liang B, Lu X, Ma Y, Li L, Wang H, Chen Z, Li Q, Gale RP (2020) COVID-
19 in persons with haematological cancers. Leukemia. 34:16371645

3. Forsyth C, Melville K, Tiley C (2018) The delayed diagnosis of myeloproliferative neoplasms is common and results in a high incidence of potentially preventable thrombotic complications. Pathology. 50:775-776

4. De Vincentiis L, Carr RA, Mariani MP, Ferrara G (2020) Cancer diagnostic rates during the 2020 "lockdown", due to COVID-19 pandemic, compared with the 2018-2019: an audit study from cellular pathology. J Clin Pathol:jclinpath-2020-206833. https://doi.org/ 10.1136/jclinpath-2020-206833

Publisher's note Springer Nature remains neutral with regard to jurisdictional claims in published maps and institutional affiliations. 